\title{
Percepción y causación: apuntes para la construcción de una crítica a la teoría de la integración conceptual de Fauconnier y Turner
}

\author{
Felipe Beltrán Vega ${ }^{* *}$
}

Recibido: 4 de septiembre de 2013

Evaluado: 20 de septiembre de 2013

Aceptado: 7 de octubre de 2013

\section{RESUMEN}

Las preguntas acerca de cómo se lleva a cabo la integración en la experiencia visual de diversos aspectos (color, forma, movimiento, etc.), procesados en áreas corticales del cerebro humano diferentes y distantes entre sí, encuentran una posible respuesta en la propuesta de Gilles Fauconnier y Mark Turner acerca de la "integración conceptual" como operación fundamental de la racionalidad humana. Esta respuesta revela, ante el análisis, cuáles son algunas de las limitaciones que el planteamiento de los autores considerados debería señalar.

Palabras clave: integración conceptual, integración perceptual, percepción visual, cognición, dación de sentido.

Artículo de investigación. Versión breve de los planteamientos centrales del trabajo de grado en curso con el mismo nombre, que se presentará para

** Master of Arts in Interactive Media, Goldsmiths College, University of London. Profesor asociado al Departamento de Humanidades de la Universidad 


\section{Perception and causation: notes for the construction of a critique of the theory of conceptual integration of Fauconnier and Turner}

\section{Abstract}

The main questions concerning the binding in experience of the diverse aspects of vision (color, shape, movement, etc.) that are processed in different and distant cortical areas of the human brain, find a possible answer in Gilles Fauconnier and Mark Turner's proposal about conceptual integration as the main cognitive operation behind human reasoning. Such a possible answer reveals upon analysis, which are some of the limits that the authors' proposal should appoint.

Keywords: conceptual integration, perceptual binding, visual perception, cognition, sense-giving.

Received: September 4, 2013

Evaluated: September 20, 2013

Accepted: October 7, 2013 


\title{
Percepção e causação: notas para a construção de uma crítica à teoria da integração conceitual de Fauconnier e Turner
}

\begin{abstract}
Resumo
Recebido: 4 de setembro de 2013

As perguntas sobre como realizar a integração na experiência visual de diversos aspectos (cor, forma, movimento, etc.), processaAceito: 7 de outubro de 2013 dos nas áreas corticais diferentes e distantes do cérebro humano, acham uma possível resposta na proposta de Gilles Fauconnier e Mark Turner em "integração conceitual", como operação fundamental da racionalidade humana. Esta resposta revela, frente à análise, quais são algumas das limitações que o planeamento dos autores apresentados deveria ser sinalado.

Palavras-chave: integração conceitual, integração percentual, percepção visual, cognição, dando sentido.
\end{abstract}




\section{INTRODUCCIÓN}

Intuitivamente, nuestra comprensión acerca de la percepción visual está anclada en una tradición de sesgo cartesiano en la que se concibe que percibir es principalmente un proceso equivalente a recibir y almacenar información proveniente del entorno. Así, describir el funcionamiento de la percepción visual se ha comprendido comúnmente como la labor de especificar la operación de un sistema que recibe una muestra de lo que nos rodea y la "codifica" para enviarla hacia otros sistemas independientes, cuyos procesos fundamentan distintas funciones cognitivas superiores, como el lenguaje, la memoria y el pensamiento. Una de las principales implicaciones de adoptar esta perspectiva es que la percepción debe comprenderse como un proceso independiente de los procesos que equivalen a lo que llamamos cognición.

Sin embargo, existe evidencia neuropsicológica y conductual (Barsalou, 1999; Mandler, 2004) que ha mostrado que al adoptar esta concepción podemos estar equivocados. Barsalou (1999) argumenta que la distinción entre la percepción y la cognición es principalmente una manifestación del supuesto común en las teorías modernas del conocimiento acerca de que las representaciones cognitivas (como aquellas que podríamos llamar conceptos) deben ser inherentemente no perceptivas. Su exposición demuestra que la percepción y la cognición bien podrían compartir sistemas de codificación de la información, tanto en el nivel neuronal como en el cognitivo. Por ello, se propone que en última instancia no hay una distinción real entre esos sistemas, sino que se configuran - ciertamente de un modo complejo- como uno solo.
Mandler (2004, p. 49), por su parte, sostiene que los conceptos - entendidos como conjuntos de conocimientos declarativos explícitos acerca de clases de cosas y de eventos que resultan potencialmente accesibles al pensamiento consciente- son diferentes y se procesan de distinta manera a cómo se codifica el conocimiento procedimental o motor. Sin embargo, muestra que los hallazgos fundacionales de las investigaciones de Piaget acerca del desarrollo cognitivo humano pueden interpretarse bajo una luz distinta, con lo cual demuestra que los conceptos no necesariamente emergen sobre la base de asociaciones de codificaciones exclusivamente sensoriales que se han desarrollado primero. Para Mandler es un error suponer que los infantes humanos pasan por una etapa de su desarrollo exclusivamente perceptual, sin poder formarse nada parecido a las conceptualizaciones del adulto hasta que alcanzan los dieciocho meses de edad. De hecho, existe evidencia acerca de procesos tempranos de análisis del sentido de lo percibido en los que se podría fundamentar el desarrollo posterior de los conceptos.

Si tenemos en cuenta estos argumentos y se acepta que la distancia entre la percepción y la cognición no es necesariamente una distinción cualitativa sobre los tipos de procesos y los tipos de información procesada, tendremos entonces que considerar la posibilidad de que la percepción cotidiana sea mucho más próxima a los procesos que podrían ser descritos desde esta perspectiva como "conceptuales" o, al menos, como "potencialmente conceptuales". ¿Cuáles son estos procesos? ¿En qué consisten? ¿Estamos justificados en describirlos como procesos conceptuales? 
Uno de los aspectos de la percepción, que podría ser un buen candidato para centrar el desarrollo de estas cuestiones, se obtiene al considerar las preguntas que emergen al tener en cuenta que la percepción (específicamente, en la modalidad visual) integra diversos aspectos de los estímulos sensibles (como el color, la forma, la textura visual, el movimiento, etc.) con estructuras cognitivas y afectivas, en una experiencia coherente, cualitativamente diferenciada y susceptible de ser descrita subjetivamente por quien percibe.

Si existe la posibilidad de considerar que estas "integraciones perceptuales" se llevan a cabo a través de procesos justificadamente caracterizados como cognitivos, podríamos tener un argumento válido para respaldar las propuestas acerca de la percepción como un proceso que no excluye aspectos cognitivos y en los que se genera el sentido de aquello que se percibe.

\section{EL PROBLEMA DE LA "LIGADURA" (BINDING PROBLEM)}

La investigación neurofisiológica de las últimas décadas ha mostrado, sobre todo en lo referente a la percepción visual, que aunque diferentes aspectos de una misma escena se codifiquen en áreas diferentes de la corteza visual, en la experiencia aparecen todas estas diferentes propiedades como caracterizando a los diversos objetos que integran cada una de las escenas como un todo coherente; esto es, las diferentes aspectos aparecen "ligados" a objetos, y estos a su vez conforman totalidades que pueden ser descritas como escenas o eventos. En este punto, el problema radica principalmente en la incapacidad para determinar cómo estos procesos de "integración perceptual" se llevan a cabo, si se tiene en consideración que no existe evidencia alguna que respalde la hipótesis de la existencia de un único centro anatómicamente identificable hacia el que pueda converger toda la información procesada "separadamente".

La dimensión más saliente de este problema, que se presenta al intentar describir cómo se logra la integración de los objetos percibidos con las ubicaciones que ocupan, se evidencia en la codificación independiente de información (activación neuronal) que recorre las vías ventral y dorsal, conocidas respectivamente como la "ruta del qué" y la "ruta del dónde o del cómo" (Treisman, 1996). La ruta dorsal se extiende desde la corteza visual primaria (V1) en el lóbulo occipital hacia el lóbulo parietal. Está asociada con funciones de ubicación y direccionamiento de la acción motriz en el espacio. La ruta ventral, por su parte, constituye un flujo de actividad neuronal que se alimenta de la actividad en la capa parvocelular del núcleo geniculado central del tálamo, cuyas células proyectan hacia el área V1 y posteriormente a través de V2 y V4 hacia áreas inferiores del lóbulo temporal. Está asociada con funciones de identificación y categorización de los objetos percibidos. A pesar de existir evidencia de múltiples puntos de interconexión entre estas vías, la manera detallada en la que se integran los tipos de información que sus actividades instancian es aún el misterio por revelar detrás de los casos documentados de patologías descritas como "cegueras cognitivas", en las que el daño causado generalmente por accidentes cardiovasculares en una $u$ otra de las vías produce cegueras selectivas en las que el sujeto no puede "ver" 
el objeto que de todas formas puede manipular hábilmente, o a la inversa: no puede ubicar espacialmente cualquiera de los objetos que perfectamente reconoce según su apariencia.

Sin embargo, la información acerca de los objetos y de sus ubicaciones no es lo único que se liga en la percepción cotidiana. Al estudiar los procesos perceptuales en mayor detalle se ha descubierto que ciertos valores específicos de dimensiones particulares de la experiencia visual son instaurados por rangos de actividad neuronal en poblaciones de neuronas distintas (Treisman, 1996). Por ejemplo, la experiencia concreta de un color como el púrpura en la dimensión cromática de la visión se explica como la activación de células detectoras, sensibles a un amplio rango de estímulos, que logran implementar esa sensación particular como una distinción fina que resulta de la combinación $-\mathrm{y}$, por supuesto, de la ligadura - de la actividad de diferentes células con sensibilidades sobrepuestas.

También al percibir integramos la información acerca de los estados sucesivos de un mismo objeto experimentado a lo largo de un intervalo de tiempo determinado, como un caso de movimiento (real o aparente) o de otras transformaciones (crecimiento, disminución de tamaño, deterioro, etc.).

Algunas propuestas que se han considerado recientemente como explicación de estos mecanismos integradores involucran diversas alternativas, entre las que se incluyen: postular la existencia de células neuronales especialmente "sintonizadas" para la detección de conjunciones de rasgos; proponer que la atención hacia un área específica del espacio percibido prefigura y afecta las condiciones de la integración; señalar que existe una activación neuronal sincronizada de células en áreas distantes, pero interconectadas, de la corteza cerebral; o incluso combinaciones diversas de todas estas diferentes alternativas.

Aunque tradicionalmente el conjunto de las preguntas que engloba este asunto ha sido tratado desde la filosofía, la psicología y las diferentes aproximaciones neuropsicológicas y neurofisiológicas, es importante reconocer adicionalmente las implicaciones semióticas que pueda tener preguntarse cómo y dónde se integra la información proveniente de diversas modalidades sensoriales, en la medida en que al plantear este tipo de preguntas se está abordando la cuestión acerca del surgimiento del sentido en la experiencia consciente (Bob, 2011). Para plantear la cuestión en términos más explícitos: si fuera posible demostrar de qué manera se integran en experiencias unificadas y coherentes las diversas modalidades de estímulos que se procesan en áreas corticales distantes, no solamente estaríamos ofreciendo una respuesta ante las preguntas sobre la integración perceptual, sino que también estaríamos ofreciendo una propuesta acerca de cómo esta integración resulta significativa como evento en la experiencia. Así, podríamos respaldar los argumentos acerca de los procesos perceptuales como procesos en los que no solamente se captura información del entorno, sino en los que también emerge el sentido de aquello que es percibido.

Es claro que ninguno de los aspectos sensoriales tiene sentido por sí solo sin aparecer integrado en una experiencia. Únicamente al integrar diversas modalidades sensoriales 
en totalidades, esos "bloques" de experiencia que podríamos llamar eventos resultan significativos; es decir, adquieren el sentido que los distingue de otros eventos similares y con el que posteriormente pueden ser subjetivamente descritos. El propio Descartes formuló una solución ante su comprensión del problema en la que el "centro de la integración" - según él ${ }^{1}$, la glándula pineal, en la que se liga la información que proviene de cada uno de los sentidos - es también el punto de convergencia entre lo corpóreo y lo mental. Es justo allí donde la actividad sensorial (del cuerpo) se transforma en experiencia subjetiva (mental).

Sin necesidad de acoger un dualismo cartesiano, podemos evidenciar que el sentido de nuestras experiencias conscientes como totalidades coherentes parece requerir de la integración de diferentes patrones de actividad neuronal que instancian el procesamiento de información de rasgos distintos del entorno. La información que capturamos del entorno es constantemente distribuida y procesada en regiones cerebrales distantes que tienen estructuras y funciones diferenciadas con relativa claridad. Por ello, el problema que se

1 Inicialmente propuso en El tratado del hombre que los patrones del movimiento de los flujos que circulan a través de los canales que rodean la glándula son la causa de que las experiencias visuales "representen en el alma" las apariencias del mundo exterior que es observado. Posteriormente, en Las pasiones del alma" formuló más concretamente su propuesta acerca de que es allí en dónde el alma ejerce directamente sus funciones sobre el cuerpo:

The part of the body in which the soul directly exercises its functions is not the heart at all, or the whole of the brain. It is rather the innermost part of the brain, which is a certain very small gland situated in the middle of the brain's substance and suspended above the passage through which the spirits in the brain's anterior cavities communicate with those in its posterior cavities. The slightest movements on the part of this gland may alter very greatly the course of these spirits, and conversely any change, however slight, taking place in the course of the spirits may do much to change the movements of the gland (AT XI, 351, CSM I, 340). plantea está en saber cómo logramos integrar esta información sin apelar a la propuesta de un centro, anatómica o funcionalmente identificable en el que converja.

\section{EL PROBLEMA DE \\ LA "LigAdURA" RESUElTO COMO PROCESO DE INTEGRACIÓN CONCEPTUAL (BINDING AS BLENDING)}

Recientemente, autores emparentados con perspectivas cognitivistas han propuesto que independientemente de las consideraciones acerca de la anatomía neuronal de la integración de "información" multimodal proveniente del entorno en una experiencia unificada y coherente, la reformulación de los límites entre los procesos de percepción y de cognición abre paso a una consideración acerca de los procesos tradicionalmente comprendidos como conceptuales, en cuanto constituyen recursos para alcanzar la integración perceptual.

Se abre entonces la posibilidad de considerar ciertos procesos - tradicionalmente comprendidos como procesos conceptualescomo parte integral de los mecanismos de integración de diversas modalidades sensoriales en experiencias unificadas y coherentes. Indagar acerca de los procesos de integración conceptual podría ofrecer respuestas potenciales ante la pregunta acerca de cómo se consigue integrar coherentemente la información proveniente de diferentes modalidades sensoriales en una única experiencia.

En este texto, revisaremos puntualmente la propuesta de Fauconnier y Turner (2002) sobre la teoría de la integración conceptual 
(conceptual blending), concebida como recurso para explicar la integración perceptual de diversas modalidades sensoriales como rasgos diferenciados que corresponden a las características de un objeto único cuando es percibido. Al hacerlo, buscaremos desarrollar la idea acerca de que la experiencia humana se constituye como una construcción permanente del sentido del entorno que nos rodea, lo que permite trazar límites más claros en cuanto a la percepción como proceso en conexión ineludible con los procesos que soportan lo que identificamos como cognición.

La teoría de la integración conceptual que se discutirá en este texto está fundamentada en la teoría de los espacios mentales de Fauconnier (1994), propuesta inicialmente para resolver cuestiones acerca de la referencia indirecta y de la "opacidad referencial" en las que el referente de una expresión es ambiguo y debe, por tanto, resolverse apelando al contexto (por ejemplo, cuando se afirma que "Juan pintó una pared" y resulta posible interpretar la frase en relación a que Juan pintó un cuadro en el que aparece un muro, o a que Juan cubrió una pared con pintura [Pascual, 2012, p. 147]). Un espacio mental puede describirse como una estructura conceptual parcial que se emplea para representar una realidad posible, tal y como es percibida, recordada o comprendida por un "ente cognoscitivo" que la activa de forma dinámica cuando escucha un discurso o cuando lee un texto (Pascual, 2012, p. 150.)

En la teoría de la integración conceptual que se presentará en lo que sigue (Fauconnier y Turner, 2002), la operación cognitiva fundamental que se "aplica" sobre los espacios mentales es un proceso de integración o de "amalgamado" que hace posible que dos (o más) espacios mentales distintos e independientes aporten algunos elementos de sus contenidos hacia un tercer espacio en el que, al integrarse, emerge un nuevo sentido. Lo que resulta particularmente llamativo para nuestros propósitos presentes es que los autores proponen que su modelo resulta útil para dar cuenta de la integración de diversas modalidades sensoriales diferentes en una experiencia coherentemente unificada, lo que hace posible considerar los procesos de integración conceptual como parte de los procesos perceptuales:

The perception of a single entity, such as a cup, is an imaginative feat still very poorly understood by neurobiologists. The perception available to consciousness is the effect of complicated interactions between the brain and the environment. But we integrate that effect with its causes to create emergent meaning: the existence of a cause - namely, the cup - that directly presents its effect - namely, its unity, color, shape, weight, and so on-. As a consequence, the effect is now in its cause: the color, shape, and weight are now intrinsically, primitively and objectively in "the cup" (Fauconnier y Turner, 2002, p. 78)

2 "La percepción de una entidad simple, como una taza, es un logro imaginativo aun muy pobremente entendido por los neurobiólogos. La percepción disponible a la conciencia es el efecto de complicadas interacciones entre el cerebro y el entorno. Pero integramos ese efecto con sus causas para crear un sentido emergente: la existencia de una causa - es decir, la taza - que directamente presenta sus efectos - es decir, su unicidad, color, forma, peso, y demás - como una consecuencia. El efecto está ahora en su causa: el color, forma y peso están intrínsecamente, primitivamente y objetivamente en la 'taza". 


\section{INTEGRACIÓN CONCEPTUAL: PRESENTACIÓN GENERAL DEL MODELO}

Describiremos el modelo que presentan Fauconnier y Turner (2002) en mayor detalle, a partir de la discusión de un ejemplo relativamente simple. Consideremos la comprensión del sentido en la frase "Las perlas de tu boca". Para empezar, imaginaremos dos o más estructuras conceptuales diferentes, caracterizadas como conjuntos de información parcial que se construye sobre la marcha (o como se dice más técnicamente, "que se actualiza en-línea") mientras hablamos o pensamos (Fauconnier y Turner, 2002, p. 102).

La información así actualizada se relaciona y se jerarquiza a partir de un conocimiento esquemático extraído de la memoria a largo plazo, la cual estructura su composición en "marcos" que, dependiendo de la ocasión, pueden presentar diferentes grados de abstracción o generalidad. Estas estructuras conceptuales serán llamadas "espacios mentales de entrada" o simplemente "espacios de entrada" (input spaces), para abreviar.

La descripción de un proceso de integración conceptual empieza típicamente por un proceso de estructuración en el que se constituyen estos espacios de entrada y se establecen ciertas conexiones entre los elementos o las relaciones en cada uno. Estas conexiones se fundamentan en la identificación de elementos estructurales comunes a los espacios de entrada, por lo que se hace posible superponer la estructura básica de un espacio de entrada sobre otro y establecer así mapeos entre estos. Se hablará de mapeos cuando en un espacio mental de entrada existan elementos equivalentes o relaciones entre elementos equivalentes con las de otro espacio mental de entrada.

En el caso de este ejemplo, cierta información acerca de las perlas y sobre la boca se construye como análoga en el interior de dos espacios mentales de entrada independientes, que están estructurados por marcos que contienen elementos identificados como análogos. Específicamente, consideraremos el "esquema de imagen" de contenedor ${ }^{3}$ que estructura la información acerca de la boca y los dientes de manera análoga a como se estructura la información acerca de las perlas y las ostras. Las perlas están en cierta relación con su contenedor, que resulta análoga a la relación en la que están las bocas con los dientes; ambos espacios de entrada han sido construidos para que ciertos elementos de las perlas se puedan integrar con ciertos elementos de los dientes como contenido análogo de las bocas.

En este caso es importante señalar que los dientes se representan como un contenido "granular" en cuanto no son un contenido unitario y continuo de la boca. Están dentro de la boca, pero cada uno puede identificarse como una unidad independiente que puede considerarse por separado con respecto a los demás. Asimismo, al abrir la ostra, las perlas no constituyen un contenido

3 En cuanto los "esquemas de imagen" emergen en las acciones corporales de manipulación y navegación en el espacio efectuadas por individuos que comparten una configuración corporal análoga, constituyen estructuras de la sensibilidad humana (modos recurrentes de percibir, orientarse e interactuar) que no se quedan en la experiencia individual de cada sujeto, sino que están influidos por las maneras en las que la comunidad a la que se pertenece interpreta y describe estos patrones generales como modos de experiencia culturalmente compartidos que configuran la comprensión del mundo como un conjunto de experiencias coherentes (Johnson, 1990, p. 14). 
unitario y continuo, sino que - al igual que los dientes en la boca - son un contenido "granular" que puede considerarse como un conjunto de unidades independientes las unas con respecto a las otras, e independientes a su vez del contenedor.

Según como hayan sido construidos los espacios mentales de entrada, se establecerán ciertas relaciones de mapeo entre estos. Las relaciones que hacen que ciertos elementos y relaciones de un espacio de entrada puedan ser comprendidas como contrapartes de elementos y relaciones de otro, se representan de forma abstracta en un tercer espacio mental llamado "espacio genérico", el cual orienta la proyección selectiva de algunos de los aspectos de la información representada en cada uno de los espacios de entrada hacia un cuarto espacio mental en el que se lleva a cabo su integración.

Dentro de la teoría de la integración conceptual de Fauconnier y Turner, algunas de las conexiones que sustentan los mapeos entre los espacios de entrada pueden concebirse como "relaciones vitales", en cuanto constituyen relaciones recurrentes con las que los humanos estamos "sintonizados" para conceptualizar el mundo (Fauconnier y Turner, 2002, p. 394).

Estas relaciones vitales pueden caracterizarse como aquellas - seleccionadas entre las conexiones que se establecen entre uno y otro de los espacios de entrada o relaciones inter-espaciales - que puedan comprimirse de forma recurrente en la integración como relaciones entre los elementos de un mismo espacio (relaciones intraespaciales). Algunas de estas relaciones recurrentemente identificables son analogía, disanalogía, cambio, identidad, tiempo, espacio, partetodo y causa-efecto.

Así, en el ejemplo, en uno de espacios de entrada, los contenidos acerca de las perlas instauran una relación a partir del esquema de imagen parte-todo que se identifica como análogo al esquema de imagen con el que se construye la relación entre los contenidos del espacio mental acerca de las bocas. Por esto, los espacios de entrada se puedan mapear entre sí, y esta relación se representa de forma abstracta en un espacio mental adicional llamado "espacio genérico", que orienta cuáles de los elementos parciales de cada uno de los espacios de entrada que serán proyectados hacia un cuarto espacio para su integración.

En mayor detalle, la construcción del sentido en la expresión "las perlas de tu boca" pasa por un proceso de "composición" en el que diferentes elementos provenientes de los espacios de entrada se componen para proporcionar relaciones que no existían por separado en ninguno de estos. En este caso, la composición es la que hace posible construir una relación de analogía entre las perlas y los dientes, aunque en ninguno de los espacios de input esté representada explícitamente esta relación. Los elementos de un espacio de entrada que tienen contrapartidas en los elementos del otro espacio se pueden componer en el "espacio de la integración" [blended space] como dos elementos por separado o, como en este caso, pueden componerse integrados al proyectarse hacia un solo elemento en el espacio de la integración. Podemos así dar cuenta de que se representen a los dientes fusionados con ciertas propiedades de las perlas (como su brillo y carácter precioso). 
En la figura 1 podemos representar lo descrito hasta ahora del proceso.

Figura 1. Espacio genérico

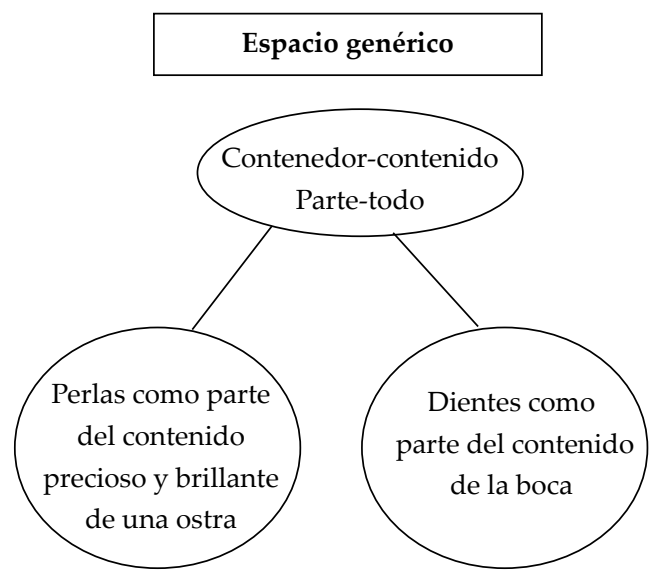

Los óvalos representan a los espacios mentales de entrada, al espacio genérico y al espacio mental integrado según como se indica. Las palabras en el interior de cada óvalo representan sus posibles contenidos. La línea horizontal que conecta a los espacios de entrada representa un mapeo entre las relaciones en cada uno. Las líneas diagonales que unen a los espacios de entrada con el espacio genérico representan la identificación de una estructura abstracta común a los espacios de entrada. Las líneas diagonales que unen a los espacios de entrada con el espacio de la integración representan la proyección selectiva de partes de sus contenidos que se integrarán.

Al llevar a cabo una integración conceptual puede identificarse un proceso de "compleción", en la medida en que se identifiquen grandes cantidades de conocimiento y de estructuras de fondo que son evocadas en una especie de proceso metonímico. En este, ante algunos pocos elementos de un marco familiar, se activan muchos otros, sin que seamos plenamente conscientes de ello (Fauconnier y Turner, 2002, p. 48).

En el ejemplo, ante la integración de las bocas y las perlas, se recluta la configuración familiar de la sonrisa con la que la integración se completa y que resulta mucho más rica en contenidos que simplemente la imagen mental de una boca con perlas en lugar de dientes. Nótese que la configuración de la sonrisa no hace parte de la información incluida inicialmente en ninguno de los espacios de entrada; sin embargo, esta información complementa de forma fluida a los elementos seleccionados desde el espacio de entrada de las perlas y desde el espacio de entrada de las bocas cuando se proyectan para su integración.

Finalmente, podremos describir un proceso de "elaboración" de la integración que se evidencia al tratarla como una simulación mental que se "corre" imaginativamente según el contexto de la información integrada. Así, en lugar de tener una boca humana que contiene perlas en vez de dientes, tenemos una sonrisa humana que revela unos dientes tan preciosos y brillantes como las perlas, y por esto podemos interpretar la expresión como un halago. Algunos elementos del espacio de entrada: "Perlas", y algunos elementos del espacio de entrada: "Boca", que han sido seleccionados y que se proyectan hacia otro espacio mental en el que se integran, se elaboran como una simulación mental en la que lo precioso y brillante de las perlas se integra con los dientes como contenido de una boca sonriente. 


\section{INTEGRACIÓN PERCEPTUAL. UNA CRÍTICA A LA TEORÍA DE LA INTEGRACIÓN CONCEPTUAL}

$\mathrm{Al}$ retomar el caso propuesto en la cita de la sección anterior, Fauconnier y Turner (2002) describen la experiencia visual consciente ante una taza como el resultado de un proceso de integración conceptual llevado a cabo sin que seamos conscientes. La presencia misma de la taza ante nosotros resulta ser una construcción cognitiva en la que diferentes dimensiones de los estímulos visuales (que según los autores son la verdadera causa objetiva de nuestra experiencia) se integran y adicionalmente se conciben como efecto (como propiedades que provienen de la existencia objetiva de la taza) (Fauconnier y Turner, 2002, p. 78).

Si consideramos detalladamente la descripción del proceso propuesto, podremos identificar dos dimensiones independientes que harán parte de la crítica. Inicialmente podremos cuestionarnos sobre la integración de las diversas modalidades del estímulo visual que nos afecta, en una experiencia visual concebida como un evento coherente e integrado: la forma, el color, la textura visual, etc., que hacen parte de nuestra experiencia ante la taza. Estos vendrían a configurarse como contenidos de espacios mentales aislados e independientes que de alguna manera compartirían elementos estructurales comunes, por lo que podrían mapearse unos sobre otros y configurar la estructura de un espacio genérico común que orientaría su integración. Es aquí donde empiezan a presentarse los problemas: es difícil suponer cómo estos espacios mentales pueden llegar a estar compuestos independientemente unos de otros. Por su parte, los espacios mentales son descritos como representaciones de aquello que concebimos: es claro que concebimos inicialmente la taza como una totalidad en nuestra experiencia. Un ejercicio de introspección revelará que solo podemos aislar en la consideración cada uno de los aspectos de la experiencia visual ante la taza si antes hemos tenido la experiencia de la taza como un todo, pero que no necesariamente concebimos en un primer momento estos aspectos como propiedades aisladas en el interior de espacios mentales independientes que deban posteriormente integrarse. Aun si esto fuera fenoménicamente posible, tendríamos que considerar cuál sería la estructura común a todos y cada uno de los espacios de entrada que contienen estas "propiedades"; estructura que permitiría orientar la selección de elementos que apuntan hacia su integración.

Podemos señalar que la teoría de la integración conceptual da cuenta de las manipulaciones cognitivas que pueden implementarse sobre estructuras mentales previamente constituidas (espacios mentales), pero que presenta una primera limitación en lo relativo a dar cuenta de su configuración. Si tenemos en consideración cuáles son los espacios mentales que la presencia de un objeto (como la taza) podría evocar, podremos darnos cuenta de que independientemente de lo que se proponga, en ningún caso se evocan espacios mentales cuyo contenido esté constituido por algún aspecto parcial de la experiencia separado de los otros. De este modo, ante la ausencia de evidencia respecto a la configuración de espacios de entrada con "propiedades" aisladas de los objetos que experimentamos, podemos empezar a cuestionar la propuesta de la integración conceptual como una descripción de los procesos de integración perceptual.

Sin embargo, la integración misma no es el único problema que la consideración de la 
propuesta en la teoría nos plantea. En la explicación que se ofrece acerca de la conexión entre causas y efectos que afecta la concepción de una relación entre la existencia de la taza y la experiencia integrada de sus rasgos, se confunde la experiencia de fenómenos causales con la concepción que pueda tenerse acerca de estos. Según se plantea, en la experiencia misma concebimos el objeto experimentado como una entidad unificada que causa nuestra experiencia de sus rasgos, pero según los autores esta concepción del objeto es el resultado de un esfuerzo cognitivo de integración en el que la existencia misma de la taza como causa de su experiencia es construida como efecto, a partir de las interacciones complejas entre diversas dimensiones de los estímulos ante los que estamos expuestos, los cuales constituyen la verdadera causa (independiente de cualquier concepción) de nuestra experiencia ante el objeto. Así, la concepción de la taza como causa de nuestra experiencia ante esta misma resultaría ser un efecto intrínsecamente experimentable de nuestra percepción; el problema está en que se ignora que la experiencia unificada de un objeto antecede fenoménicamente a la concepción de su presencia como fundamento causal de nuestras experiencias ante este. Percibimos objetos como una integración coherente de sus rasgos y eventualmente podemos concebirlos como causas de nuestras experiencias ante ellos; no a la inversa como se plantea en la teoría.

La integración perceptual de los rasgos procesados independientemente en una experiencia unificada y coherente parece escapar a los límites de las construcciones de sentido que la propuesta en la teoría de la integración conceptual pretende abarcar. La teoría puede eficientemente dar cuenta de los procesos en los que los contenidos de espacios mentales distintos se integran para construir un sentido nuevo, pero no puede dar cuenta de la configuración de estos espacios como representaciones acerca de la experiencia coherentemente unificadas. En lo que respecta a los alcances de la propuesta en consideración, la integración perceptual que caracteriza nuestra experiencia cotidiana no es igual a las demás integraciones que pretende abarcar la teoría. La integración perceptual es un hecho que fundamenta la posibilidad de construcción de los espacios mentales como los que pueden integrarse según como se describe en la teoría. Pero esos mismos procesos no dan cuenta de cómo esta experiencia se unifica.

\section{Referencias}

Barsalou, W. L. (1999). Perceptual symbol systems. Behavioral and Brain Sciences, 22, 577-660.

Bob, P. (2011). Brain, mind and consciousness: Advances in neuroscience research. Nueva York: Springer.

Fauconnier, G. (1994). Mental spaces: aspects of meaning construction in natural language. Cambridge: Cambridge University Press.

Fauconnier, G. y Turner, M. (2002). The way we think: Conceptual blending and the mind's hidden complexities. Nueva York: Basic Books.

Johnson, M. (1990). The body in the mind: The bodily Basis of Meaning, Imagination and Reason. Chicago: University of Chicago.

Mandler, J. M. (2004). The foundations of mind: origins of conceptual thought. Oxford: Oxford University Press.

Pascual, E. (2012). Los espacios mentales y la integración conceptual. En Ibarretxe y Valenzuela (Dirs.). Lingüística cognitiva. Barcelona: Anthropos.

Treisman, A. (1996). The binding problem. Current Opinion in Neurobiology, 6, 171-178. 